\title{
THE FLORISTIC COMPOSITION AND BIOLOGICAL SPECTRUM OF VEGETATION IN THE MEYMEH REGION OF NORTHERN ISFAHAN PROVINCE, IRAN
}

\author{
KARGAR CHIGANI, H. ${ }^{1}-$ AKBAR JAVADI, S. ${ }^{1 *}-$ ZAHEDI AMIRI, G. ${ }^{2}-$ JAFARI, ${ }^{2}{ }^{2}-$ \\ JAMALEDDIN KHAJEDDIN, S. $^{3}$ \\ ${ }^{I}$ Department of Range Management, Science and Research Branch, Islamic Azad University, \\ Daneshgah Blvd, Simon Bulivar Blvd, Tehran, C.P. 1477893855, Iran \\ (phone: +98-9131298514; fax: +98-2144868550-55) \\ ${ }^{2}$ Faculty of Natural Resources, University of Tehran, Karaj, Iran, \\ (phone: +98261 2223044-6; fax: +98261 2227765) \\ ${ }^{3}$ Range and Watershed Management Division, Department of Natural Resources, \\ Isfahan University of Technology, Isfahan, Iran \\ (phone: +98-9131104624; fax: +98-2144640229) \\ *Corresponding author \\ e-mail: hadi_k1360@yahoo.com; phone: +98-9122443659; fax: +98-2144868550-55 \\ (Received 27 ${ }^{\text {th }}$ Aug 2016; accepted 29 $9^{\text {th }}$ Oct 2016)
}

\begin{abstract}
This research details the flora of Meymeh, a region situated in the province of Isfahan in the central plateau of Iran and entirely within the Irano-Turanian phytogeographical region. In total, 164 species of flora belonging to 32 families and 108 genera were surveyed and identified in several field trips during the study period from 2013 to 2014. Based on the number of species, Asteraceae (30 species, $18.3 \%$ ), Poaceae (20 species, 12.2\%), and Papilionaceae (19 species, $11.6 \%$ ) were the most important families. The largest genera were Astragalus (15 species), Cousinia (6 species), Acanthophyllum, and Centaurea (each with 5 species). In this study, the life-form spectra were classified on the basis of Raunkiaer's system. Then, the $\chi^{2}$ test and correlation analysis were used to compare the biological spectrum with Raunkiaer's normal spectrum and with those in other floristic studies conducted in the Irano-Turanian growth zone. The results showed that the life-form spectrum in the present study was characteristic of a cool steppe climate region and dominated by hemicryptophytes (50\% of the recorded species), followed by chamaephytes and therophytes. Findings also indicated that the effects of climate, altitude, and human activities such as overgrazing caused a reduction in phanerophytes from around 46 to $4 \%$, increases in chamaephytes from about 9 to $26 \%$ and hemicryptophytes from 26 to $50 \%$ in comparison with Raunkiaer's normal spectrum. It can be concluded that hemicryptophytes and phanerophytes usually comprise the highest and lowest percentages of life forms in studies conducted in the Irano-Turanian growth zone.
\end{abstract}

Keywords: flora; life form; endangered species; Irano-Turanian; biodiversity; ecology

\section{Introduction}

Sustainable development requires the acquisition of basic information on terrestrial ecosystems. A major section of an ecosystem is vegetation where plant communities and species spread based on environmental factors (Nimais, 1985). Humans and most other animals directly and indirectly depend almost totally upon plants. Mismanagement and overexploitation have resulted in critical conditions and the degradation of vegetation. Therefore, understanding the distribution of plant species (floristic studies) is essential to the management and conservation of these ecosystems. In addition, flora studies of each region, including the list of species, the life-form spectrum, geographical 
distribution, and the identification of threatened species is useful for ecological issues like biodiversity and determining growth capacities and potentials of a region. A life form is a group of plants which have the same general morphological features (Cain, 1950). Generally, plants are understood to be a growth form that displays an obvious relationship with key environmental factors (Mueller-Dombois et al., 1974). Some techniques for categorizing plant life form have been developed, among which Raunkiaer's system is still the simplest and, in many ways, the most satisfactory classifier of plant life forms (Asri, 2003). Raunkiaer (1934) provided this classification system based on the position and degree of protection of the renewing buds which are responsible for the renewal of the aerial plant body after an unfavorable season. Accordingly, plant species can be classified into five main classes: phanerophytes, chamaephytes, hemicryptophytes, cryptophytes and therophytes.

Identification of plant species, especially rare and threatened ones, is very important to the management, protection, reclamation, and development of natural ecosystems throughout the world. The International Union for Conservation of Nature and Natural Resources (IUCN) was formed to identify plant species and take the necessary measures to prevent their extinction (IUCN, 1981). In Iran, Jalili and Jamzad (1999) attempted to introduce species based on the IUCN criteria in the "Red Data Book of Iran." Accordingly, the threatened species of Iran include four categories: endangered, vulnerable, low risk, and data deficient.

Iran has one of the richest floras with a large number of endemic species (Zohary, 1963). A significant proportion of the species are belonging to the Irano-Turanian vegetation zone. The Irano-Turanian flora and vegetation is known by the following features (Djamali et al., 2012): (i) high species richness, (ii) high diversity, (iii) scarcity of forest vegetation, (iv) high endemism (exceeding 25\%), (v) dominance of chamaephytes (mainly dwarf shrubs) and hemicryptophytes (mainly Poaceae), forming steppe vegetation, and (vi) development of several specific taxonomic groups, including the genera Astragalus (Fabaceae), Cousinia and Centaurea (Asteraceae), and Acantholimon (Plumbaginaceae).

Iran's rich and diverse flora have been studied by foreign (Zohary, 1973; Rechinger, 1963-2010) and Iranian botanists (Mobayen, 1975-1996; Ghahraman, 1975-2005) for many years. Sources report that the number of plant species in Iran is around 9500-8500 which, in comparison, is near the total number of species in Europe (Akhani, 2006). The Meymeh region in the central plateau of Iran is an example of a cold steppe region with typical steppe plants. Part of the area is located in the Karkas Mountains, and species diversity there is high because of topographic and climatic conditions. The particular climate condition, drought, over-grazing, human activity, and the reduction in valuable species in the area provide enough reasons to study the flora of the region. There are no published studies regarding the vegetation of the area. Therefore, the main objectives of the present study were to enrich the knowledge of vegetation in the area by identifying total plant species and their life-form spectrum, life span, growth habits, and geographical distribution and too identify threatened species. In addition, the study can be very useful for bridging botanical gaps in Iran. Since the climate of the area is classified as Cold Mountain, it could be expected to find a high proportion of Hemicryptophytes. Also, because this area is an indicator of the cold steppe region in central Iran, it can be expected that a high percentage of species will be related to the Irano-Turanian growth zone. In this research, the following questions were asked: (i) What is the floristic composition and life-form spectrum of the study area? (ii) Which 
life-form classes are significantly different from Raunkiaer's normal spectrum? (iii) Are the identified floristic spectra the same as those in other studies conducted in the IranoTuranian growth zone?

\section{Materials and methods}

\section{Study area}

This study was conducted in the Meymeh region, located north of Isfahan in the central plateau of Iran (Fig. 1). The study area occupying approximately $83.4 \mathrm{~km}^{2}$ is located between latitudes $33^{\circ} 20^{\prime}$ to $33^{\circ} 41^{\prime} \mathrm{N}$ and longitudes $51^{\circ} 6^{\prime}$ to $51^{\circ} 33^{\prime} \mathrm{E}$ and the elevation ranges between 2004 and $3157 \mathrm{~m}$ and the average slope is $18 \%$. The annual precipitation of the study area influenced mainly by the Mediterranean atmospheric system is about $177 \mathrm{~mm}$ with a mean annual temperature of about $12{ }^{\circ} \mathrm{C}$. Most rainfall (73\%) was concentrated between November and May. The climate of the study area was, following Emberger method, cold-arid and following Köppen-Geiger climate classification system was cold steppe (cold semi-arid) climate with average annual temperature below $18^{\circ} \mathrm{C}$ 'Bsk'.

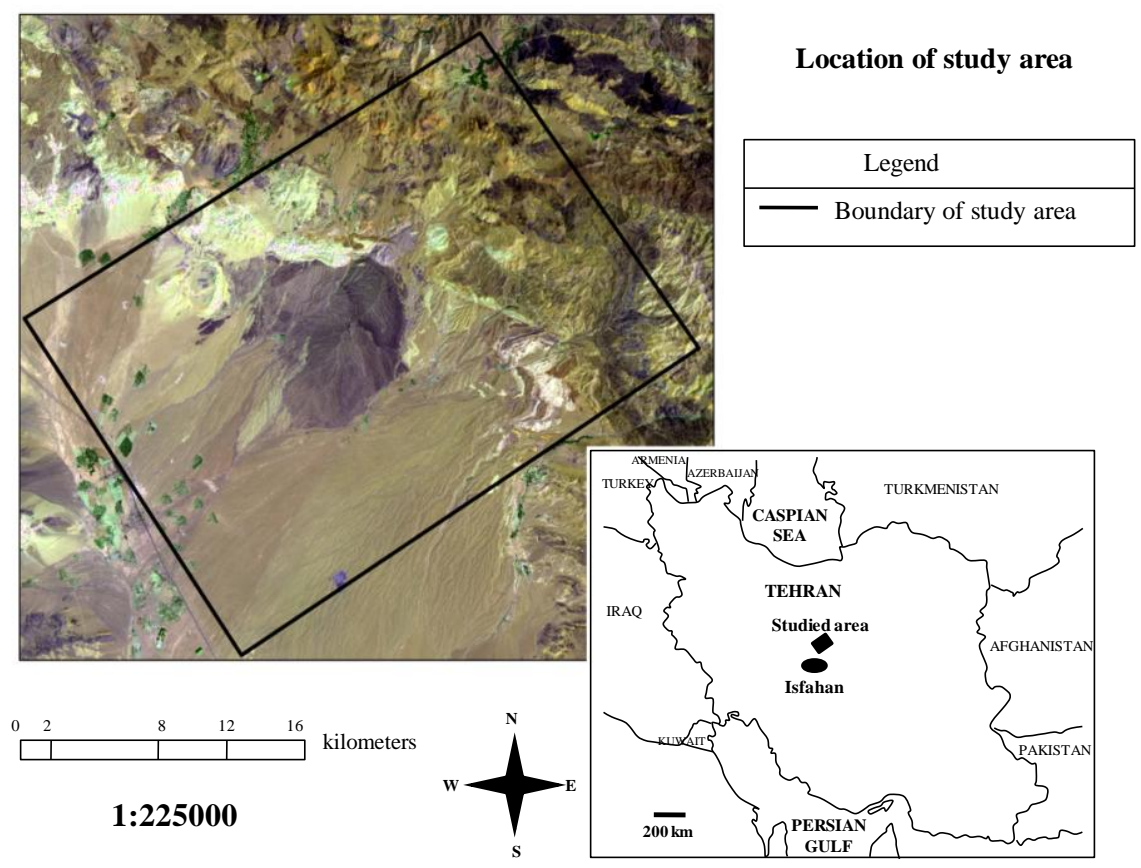

Figure 1. Location of the study area.

\section{Data collection and statistical analysis}

The flora was surveyed and identified in different seasons on several field trips taken during the period 2014 to 2015 . The specimens collected were prepared according to standard herbarium techniques and recognized according to the Flora Iranica (Rechinger, 1963-2010), Flora of Turkey (Davis, 1965-1988), Flora of Iran (Mobayen, 1975-1996), Astragalus communities of Iran (Ramak Masomi, 1986-2000) Colored Flora of Iran (Ghahraman, 1975-2005), Flora of Iran (Assadi et al., 1988-2011), and the number of the available papers (Abbasi et al., 2012; Khajeddin et al., 2012). The 
number of genera in each family and the number of species in each genus were determined using "The Plant Book" (Mabberley, 2008). All species were classified into annuals and perennials according to life spans and into herbs, shrubs, and trees according to their growth habits. The life forms of the identified species were assigned on the basis of definitions by Raunkiaer (1934). The geographical distribution and endemism of plant species were also determined according to mentioned Flora and "Biodiversity of Plant Species in Iran" (Ghahreman and Attar, 1998). The proportion of species in each life-form class (biological spectrum) was compared with Raunkiaer's normal spectrum using a $\chi^{2}$ test (Moradi et al., 2010). Then, a pairwise comparison was made of the floristic biological spectra of the study and those in other floristic studies conducted in the Irano-Turanian growth zone in the vicinity of the area by using $\chi^{2}$ tests and correlation analyses. The conservation status of the various species was evaluated using a series of criteria such as life span, geographical distribution, life form, population size, and the reproduction of plants in their natural habitats as well as the exploitation of the plant by humans, livestock, and wildlife. Finally, the threatened species in the area were classified on the basis of the method described by Jalili and Jamzad (1999).

\section{Results}

\section{Floristic composition}

In total, 164 species out of 108 genera and 32 families were collected and identified (Table 1). In this plant collection, the families Asteraceae, Poaceae, and Papilionaceae were treated as the largest plant families respectively by allocating $18.3 \%$ (30 species), $12.2 \%$ (20 species), and 11.6\% (19 species) of all the available species in the region (Fig. 2). The genera with the highest species richness were Astragalus (with 15 species), Cousinia (with 6 species), Acanthophyllum and Centaurea (each with 5 species). Annuals and perennials accounted for 17\% (28 species) and 83\% (136 species) of total number of plant species, respectively. The evaluation of growth habits showed that 124 species (83\%) made up a high proportion of the herb form, 20.7\% (34 species) belonged to the shrub group, and $3.7 \%$ (6 species) belonged to tree species.

Table 1. List of species, families and their life-form, growth habit, life span, and geographical distribution in Meymeh region, Abbreviations used in this article: $\mathrm{He}=$ Hemicryptophyte, $\mathrm{Ch}=$ Chamaephyte, $\mathrm{Cr}=$ Cryptophyte, Th= Therophyte, $\mathrm{Ph}=$ Phanerophyte (Raunkiaer, 1934), IT = Irano-Turanian, $M=$ Mediterranean, ES= EuroSiberian, Cosm $=$ Cosmopolitan, SA $=$ Saharo-Arabian, $S S=$ Sahara-Sendiananan, Plur= Pluriregional, End= Endemic (Rechinger, 1963-2010), Vu=Vulnerable, LR=Lower risk (Jalili and Jamzad, 1999), A= Annual, $P=$ Perennial, $H=$ Herb, $S=$ Shrub, $T=$ Tree.

\begin{tabular}{l|l|c|c|c|c|c}
\hline & SCIENTIFIC NAME & $\begin{array}{c}\text { Life } \\
\text { form }\end{array}$ & Chorotype & $\begin{array}{c}\text { Threatened } \\
\text { species }\end{array}$ & $\begin{array}{c}\text { Growth } \\
\text { habit }\end{array}$ & $\begin{array}{c}\text { Life } \\
\text { Span }\end{array}$ \\
\hline & Apiaceae & & & & & \\
2 & Echinophora platyloba DC. & $\mathrm{He}$ & IT (End.) & $\mathrm{LR}$ & $\mathrm{H}$ & $\mathrm{P}$ \\
3 & Eryngium billardieri F. Delaroche & $\mathrm{He}$ & IT, ES & - & $\mathrm{H}$ & $\mathrm{P}$ \\
4 & Eryngium bungei Boiss. & $\mathrm{He}$ & IT (End.) & - & $\mathrm{H}$ & $\mathrm{P}$ \\
& Ferula ovina (Boiss.) Boiss. & $\mathrm{He}$ & $\mathrm{IT}$ & $\mathrm{Vu}$ & $\mathrm{H}$ & $\mathrm{P}$ \\
5 & Asteraceae & & & & & \\
6 & Achillea wilhelmsii C. Koch & He & IT, ES & - & $\mathrm{H}$ & $\mathrm{P}$ \\
7 & Artemisia aucheri Boiss. & $\mathrm{Ch}$ & $\mathrm{IT}$ & - & $\mathrm{S}$ & $\mathrm{P}$ \\
& Artemisia sieberi Besser. & $\mathrm{Ch}$ & IT, ES & - & $\mathrm{S}$ & $\mathrm{P}$
\end{tabular}


Centaurea albonitens TURRILL

Centaurea gaubae (Bornm.) Wagenitz

Centaurea lurestanica

Centaurea ispahanica Boiss.

Centaurea virgata Lam.

Cirsium congestum Fisch. \& C.Amey. Ex

Cousinia bachtiarica Boiss \& Hausskn.

Cousinia cungesta

Cousinia lactiflora Rech. f.

Cousinia lasiolepis Boiss

Cousinia multiloba DC.

Cousinia piptocephala Bunge

Echinops caphaletes

Echinops elymaticus Born

Echinops robustus Bunge.

Gundelia tournefortii L.

Hertia angustifolia (DC.) O. Kuntze

Lactuca glauciifolia Boiss.

Lunea spinosa

Onopordon heteracanthum C. A. Mey.

Outreya carduiformis Jaub. \& Spach

Scariola orientalis (Boiss.) Sojak

Scorzonera mucida Rech.f., Aellen \&

Scorzonera tortuosissima Boiss.

Tanacetum polycephalum Schultz-Bip.

Taraxacum bessarabicum Fisch

Trapopogon caricifolius Boiss

\section{Boraginaceae}

Lappula microcarpa (Ledeb.) Gurke

Onosma elwendicum Wettst.

Rochelia macrocalyx Bunge

Brassicaceae

Alyssum heterotrichum Boiss

Alyssum linifolium Stephan ex Willd.

Alyssum bracteatum Boiss. \& Buhse

Alyssum szovitsianum Fisch. \& C.A.Mey

Barbarea vulgaris-Beloris vulgaris

Clypeola dichotoma Boiss.

Descurainia sophia (L.) Schur.

Isatis cappodocica Desv.

Lepidium persicum Boiss.

Matthiola alyssifolia (DC.) Bornm.

Matthiola ovatifolia (Boiss.) Boiss.

Moriera spinosa Boiss.

Sisymbrium irio L.

\section{Capparidaceae}

\section{Capparis spinosa L.}

Cleome coluteoides Boiss.

\section{Caprifoliaceae}

Lonicera nummulariifolia Jaub. \& Spach

\section{Caryophyllaceae}

Acanthophyllum bracteatum Boiss.

Acanthophyllum crassifolium Boiss

Acanthophyllum microcephalum Boiss.

Acanthophyllum squarrosum Bioss

Acanthophyllu spinosum

Buffonia macrocarpa Ser.

Dianthus orientalis Adams

Dianthus crinitus Sm.

\begin{tabular}{|c|c|c|c|}
\hline $\mathrm{He}$ & IT, ES & LR & $\mathrm{H}$ \\
\hline $\mathrm{He}$ & IT (End.) & LR & $\mathrm{H}$ \\
\hline $\mathrm{He}$ & IT (End.) & - & $\mathrm{H}$ \\
\hline $\mathrm{He}$ & IT (End.) & - & $\mathrm{H}$ \\
\hline $\mathrm{He}$ & $\mathrm{IT}, \mathrm{ES}$ & - & $\mathrm{H}$ \\
\hline $\mathrm{He}$ & IT, M & - & $\mathrm{H}$ \\
\hline $\mathrm{He}$ & IT (End.) & - & $\mathrm{H}$ \\
\hline $\mathrm{He}$ & IT (End.) & - & $\mathrm{H}$ \\
\hline $\mathrm{He}$ & IT (End.) & - & $\mathrm{H}$ \\
\hline $\mathrm{He}$ & IT & - & $\mathrm{H}$ \\
\hline $\mathrm{He}$ & IT (End.) & - & $\mathrm{H}$ \\
\hline $\mathrm{He}$ & IT (End.) & $\mathrm{Vu}$ & $\mathrm{H}$ \\
\hline $\mathrm{He}$ & IT & - & $\mathrm{H}$ \\
\hline $\mathrm{He}$ & IT (End.) & - & $\mathrm{H}$ \\
\hline $\mathrm{He}$ & IT (End.) & - & $\mathrm{H}$ \\
\hline $\mathrm{Ch}$ & $\mathrm{IT}, \mathrm{M}$ & - & $\mathrm{H}$ \\
\hline $\mathrm{Ch}$ & IT & LR & $\mathrm{S}$ \\
\hline $\mathrm{Th}$ & IT & - & $\mathrm{H}$ \\
\hline $\mathrm{He}$ & IT & - & $\mathrm{H}$ \\
\hline $\mathrm{He}$ & IT & - & $\mathrm{S}$ \\
\hline $\mathrm{He}$ & IT & - & $S$ \\
\hline $\mathrm{Ch}$ & IT & - & $\mathrm{H}$ \\
\hline $\mathrm{He}$ & IT (End.) & LR & $\mathrm{H}$ \\
\hline $\mathrm{He}$ & IT (End.) & - & $\mathrm{H}$ \\
\hline $\mathrm{He}$ & IT, ES & LR & $\mathrm{H}$ \\
\hline $\mathrm{He}$ & IT & - & $\mathrm{H}$ \\
\hline $\mathrm{He}$ & IT (End.) & - & $\mathrm{H}$ \\
\hline Th & IT, ES & - & $\mathrm{H}$ \\
\hline $\mathrm{He}$ & IT & - & $\mathrm{H}$ \\
\hline Th & IT & - & $\mathrm{H}$ \\
\hline Th & IT, ES & - & $\mathrm{H}$ \\
\hline $\mathrm{Th}$ & IT, M & - & $\mathrm{H}$ \\
\hline $\mathrm{He}$ & IT (End.) & - & $\mathrm{H}$ \\
\hline $\mathrm{Th}$ & IT & - & $\mathrm{H}$ \\
\hline $\mathrm{Ph}$ & - & - & $\mathrm{T}$ \\
\hline Th & IT & - & $\mathrm{H}$ \\
\hline $\mathrm{Th}$ & Cosm & - & $\mathrm{H}$ \\
\hline $\mathrm{He}$ & IT & $\mathrm{Vu}$ & $\mathrm{H}$ \\
\hline $\mathrm{He}$ & IT, M & - & $\mathrm{H}$ \\
\hline $\mathrm{He}$ & - & - & $\mathrm{H}$ \\
\hline $\mathrm{He}$ & IT (End.) & LR & $\mathrm{H}$ \\
\hline $\mathrm{He}$ & IT (End.) & - & $\mathrm{H}$ \\
\hline Th & IT & - & $\mathrm{H}$ \\
\hline $\mathrm{Ch}$ & IT,M,SS & - & $\mathrm{H}$ \\
\hline $\mathrm{He}$ & - & - & $\mathrm{H}$ \\
\hline $\mathrm{Ph}$ & IT, M & - & $\mathrm{T}$ \\
\hline $\mathrm{Ch}$ & IT (End.) & - & $\mathrm{S}$ \\
\hline $\mathrm{Ch}$ & IT & LR & $\mathrm{S}$ \\
\hline $\mathrm{Ch}$ & IT & - & $\mathrm{S}$ \\
\hline $\mathrm{Ch}$ & IT (End.) & - & $\mathrm{S}$ \\
\hline $\mathrm{Ch}$ & IT & - & $\mathrm{S}$ \\
\hline $\mathrm{He}$ & IT (End.) & LR & $\mathrm{H}$ \\
\hline $\mathrm{Ch}$ & IT & - & S \\
\hline $\mathrm{He}$ & IT & - & $\mathrm{H}$ \\
\hline
\end{tabular}


Gypsophila acantholimoides Bornm

Gypsophila virgata

Silene commelinifolia Boiss.

\section{Chenopodiaceae}

Anabasis aphylla L.

Eurotia ceratoides (L.) C. A. Mey.

Kochia prostrate Roth.

Kochia stellaris Moq.

Noaea mucronata (Forsk.) Aschers. Et

Salsola tomentosa (Moq.) Spach

\section{Convolvulaceae}

Convolvulus fruticosus Pall.

\section{Cyperaceae}

Carex stenophylla L.

\section{Euphorbiaceae}

Euphorbia cheiradenia

Euphorbia decipiens Boiss. \& Buhse

Euphorbia heteradena Jaub. \& Spach

Iridaceae

Iris songarica Schrenk

Ixillarion tataricum

\section{Lamiaceae}

Eremostachys macrophylla Montbret \& Aucher

Marrubium crassidens Boiss.

Mentha longifolia (L.) Huds.

Nepeta oxyodonta Boiss

Nepeta persica Boiss.

Phlomis olivieri Benth.

Salvia macrosiphon Boiss.

Salvia eremophila Boiss.

Scutellaria multicaulis Boiss. subsp.

Stachys inflata Benth.

Teucrium orientale L.

Thymus daenensis Celak

Zataria multiflora Boiss.

Ziziphora tenuior $\mathrm{L}$.

\section{Liliaceae}

Eremurus spectabilis

Tulipa biflora Pall

\section{Malvaceae}

Alcea aucheri (Boiss.) Alef.

Malva silvestris

\section{Orobanchaceae}

Orobanche alba Stephan

papaveraceae

Glusium oxylobum

Hypecoum pendulum $\mathrm{L}$.

Papaver macrostomum

Roemeria hybrida (L.) DC.

Papilionaceae

Alhagi camelorum Fisch.

Astragalus callistachys Boiss. et Buhse

Astragalus campylathus Boiss.

Astragalus fisheri

Astragalus globiflorus Bioiss

Astragalus glucocanthus Fisch.

Astragalus glumaceus

Astragalus gossypinus Fisch

Astragalus hamosus

\begin{tabular}{|c|c|c|c|}
\hline $\mathrm{Ch}$ & IT (End.) & LR & $\mathrm{S}$ \\
\hline $\mathrm{Ch}$ & IT & LR & $\mathrm{H}$ \\
\hline $\mathrm{He}$ & IT & - & $\mathrm{H}$ \\
\hline $\mathrm{He}$ & IT & - & $\mathrm{H}$ \\
\hline $\mathrm{Ch}$ & IT & - & $\mathrm{S}$ \\
\hline $\mathrm{Ch}$ & IT & - & $S$ \\
\hline Th & IT & - & $\mathrm{H}$ \\
\hline $\mathrm{Ch}$ & $\mathrm{IT}, \mathrm{M}$ & - & $\mathrm{S}$ \\
\hline $\mathrm{He}$ & IT & - & $\mathrm{H}$ \\
\hline $\mathrm{Ch}$ & IT & - & $\mathrm{H}$ \\
\hline $\mathrm{Cr}$ & IT & - & $\mathrm{H}$ \\
\hline $\mathrm{He}$ & IT & - & $\mathrm{H}$ \\
\hline $\mathrm{He}$ & IT (End.) & LR & $\mathrm{H}$ \\
\hline $\mathrm{He}$ & IT & - & $\mathrm{H}$ \\
\hline $\mathrm{Cr}$ & IT & - & $\mathrm{H}$ \\
\hline $\mathrm{He}$ & IT & - & $\mathrm{H}$ \\
\hline $\mathrm{He}$ & IT & - & $\mathrm{H}$ \\
\hline $\mathrm{He}$ & IT & - & $\mathrm{H}$ \\
\hline $\mathrm{He}$ & IT, SS, & LR & $\mathrm{H}$ \\
\hline $\mathrm{He}$ & IT (End.) & LR & $\mathrm{H}$ \\
\hline $\mathrm{He}$ & IT (End.) & - & $\mathrm{H}$ \\
\hline $\mathrm{He}$ & IT & - & $\mathrm{H}$ \\
\hline $\mathrm{He}$ & IT & - & $\mathrm{H}$ \\
\hline $\mathrm{He}$ & IT (End.) & $\mathrm{Vu}$ & $\mathrm{H}$ \\
\hline $\mathrm{He}$ & IT (End.) & LR & $\mathrm{H}$ \\
\hline $\mathrm{He}$ & IT, ES & - & $\mathrm{H}$ \\
\hline $\mathrm{He}$ & IT & - & $\mathrm{H}$ \\
\hline $\mathrm{Ch}$ & IT (End.) & LR & $\mathrm{H}$ \\
\hline $\mathrm{Ch}$ & IT (End.) & $\mathrm{Vu}$ & $\mathrm{H}$ \\
\hline Th & IT, ES & - & $\mathrm{H}$ \\
\hline $\mathrm{Cr}$ & IT & - & $\mathrm{H}$ \\
\hline $\mathrm{Cr}$ & IT & - & $\mathrm{H}$ \\
\hline $\mathrm{He}$ & - & - & $\mathrm{H}$ \\
\hline $\mathrm{He}$ & IT & - & $\mathrm{H}$ \\
\hline $\mathrm{Cr}$ & - & - & $\mathrm{H}$ \\
\hline $\mathrm{Cr}$ & - & - & $\mathrm{H}$ \\
\hline Th & IT, ES & - & $\mathrm{H}$ \\
\hline Th & IT (End.) & - & $\mathrm{H}$ \\
\hline Th & IT & - & $\mathrm{H}$ \\
\hline $\mathrm{He}$ & IT & - & $\mathrm{H}$ \\
\hline $\mathrm{Ch}$ & IT & LR & $\mathrm{S}$ \\
\hline $\mathrm{Ch}$ & IT & LR & $S$ \\
\hline $\mathrm{Ch}$ & IT & - & $\mathrm{S}$ \\
\hline $\mathrm{Ch}$ & IT & - & $\mathrm{S}$ \\
\hline $\mathrm{Ch}$ & IT & LR & $S$ \\
\hline $\mathrm{Ch}$ & IT & - & $\mathrm{S}$ \\
\hline $\mathrm{Ch}$ & IT & - & $\mathrm{S}$ \\
\hline $\mathrm{Ch}$ & IT & - & $\mathrm{S}$ \\
\hline
\end{tabular}


Astragalus microphysa Boiss. Astragalus piptocephalus Boiss Astragalus podolobus Bioiss. \& Hohen Astragalus pycnocephalus Fischer Astragalus schistosus Boiss.\&Hohen Astragalus scleroclodus Astragalus verus Boiss \& Hausskn. Onobrychis cornuta (L.) Desv. Onobrychis melanotricuma Boiss Trigonella monantha C.A. Mey. Phyllanthaceae

Andrachne telephioides $\mathrm{L}$.

\section{Plantaginaceae}

Plantago major $\mathrm{L}$. Plumbaginaceae

Acantholimon aspadanum Bunge Acantholimon festocaceum (Jaub. at Sp.) Acantholimon scorpius (Jaub. \& Spach) Acantholimon oliganthum Boiss

Poaceae

Agropyrom intermedium (Host) P. Agropyrom tauri Boiss. \& Bal.

Boissiera squarrosa Hochst. ex Steud Bromus danthoniae Trin. ex C.A.Mey. Bromus tectorum $\mathrm{L}$.

Bromus tomentellus Boiss.

Cynodon dactylon (L.) Pers.

Dactylis glomerata $\mathrm{L}$.

Eremopoa persica (Trin.) Roshev. Eremopyrum bonaepartis (Spreng.)

Eremopyrum distans (K.Koch) Nevski

Hordeum bulbosum $\mathrm{L}$.

Hordeum vulgare $\mathrm{L}$.

Lolium rigidum Gaudin

Melica persica Kunth

Oryzopsis holciformis (M.B.) Hack

Poa bulbosa L.

Poa sinaica Steud.

Stipa barbata Defs

Stipagrostis plumosa Munro ex

Polygonaceae

Atraphaxis spinosa L.

Ranunculaceae

Adonis aestivalis $\mathrm{L}$.

Thalictrum minus $\mathrm{L}$.

\section{Resedaceae}

Reseda buhseana Mull.Arg

Reseda lutea $\mathrm{L}$.

Rosaceae

Amygdalus scoparia Spach

Poterium sanguisorba $\mathrm{L}$.

\section{Rubiaceae}

Gaillonia bruguieri A.Rich. ex DC.

Rubia florida Boiss.

\section{Scrophulariaceae}

Scrophularia leucoclada Bunge

Scrophularia striata Boiss.

Verbascum songaricum Schrenk

Verbascum speciosum Schrad.

\begin{tabular}{|c|c|c|c|}
\hline $\mathrm{Ch}$ & IT & - & S \\
\hline $\mathrm{Ch}$ & IT & - & $\mathrm{S}$ \\
\hline $\mathrm{Ch}$ & IT & - & $\mathrm{S}$ \\
\hline $\mathrm{Ch}$ & IT & - & $\mathrm{S}$ \\
\hline $\mathrm{He}$ & IT & $\mathrm{Vu}$ & $\mathrm{H}$ \\
\hline $\mathrm{Ch}$ & IT & $\mathrm{Vu}$ & $\mathrm{S}$ \\
\hline $\mathrm{Ch}$ & IT & LR & $\mathrm{S}$ \\
\hline $\mathrm{Ch}$ & IT & - & $\mathrm{S}$ \\
\hline $\mathrm{He}$ & IT & - & $\mathrm{H}$ \\
\hline Th & IT & - & $\mathrm{H}$ \\
\hline $\mathrm{He}$ & IT, M & - & $\mathrm{H}$ \\
\hline $\mathrm{He}$ & - & - & $\mathrm{H}$ \\
\hline $\mathrm{Ch}$ & IT (End.) & - & $\mathrm{S}$ \\
\hline $\mathrm{Ch}$ & IT & - & $\mathrm{S}$ \\
\hline $\mathrm{Ch}$ & IT (End.) & LR & $\mathrm{S}$ \\
\hline $\mathrm{Ch}$ & IT & - & $\mathrm{S}$ \\
\hline $\mathrm{He}$ & - & - & $\mathrm{H}$ \\
\hline $\mathrm{He}$ & - & - & $\mathrm{H}$ \\
\hline Th & IT, ES, M & - & $\mathrm{H}$ \\
\hline Th & IT & - & $\mathrm{H}$ \\
\hline Th & IT, ES, M & - & $\mathrm{H}$ \\
\hline $\mathrm{He}$ & IT, ES & $\mathrm{Vu}$ & $\mathrm{H}$ \\
\hline $\mathrm{Cr}$ & Cosm & - & $\mathrm{H}$ \\
\hline $\mathrm{He}$ & IT, ES, M & - & $\mathrm{H}$ \\
\hline Th & IT, M & - & $\mathrm{H}$ \\
\hline $\mathrm{Th}$ & IT & - & $\mathrm{H}$ \\
\hline Th & IT, ES & - & $\mathrm{H}$ \\
\hline $\mathrm{Cr}$ & IT & - & $\mathrm{H}$ \\
\hline Th & IT & - & $\mathrm{H}$ \\
\hline $\mathrm{He}$ & IT & - & $\mathrm{H}$ \\
\hline $\mathrm{He}$ & IT (End.) & LR & $\mathrm{H}$ \\
\hline $\mathrm{He}$ & IT & - & $\mathrm{H}$ \\
\hline $\mathrm{Cr}$ & IT, ES & - & $\mathrm{H}$ \\
\hline $\mathrm{He}$ & IT, SA & - & $\mathrm{H}$ \\
\hline $\mathrm{He}$ & IT, ES & - & $\mathrm{H}$ \\
\hline $\mathrm{He}$ & IT & - & $\mathrm{H}$ \\
\hline $\mathrm{Ph}$ & IT & - & $\mathrm{T}$ \\
\hline Th & IT & - & $\mathrm{H}$ \\
\hline $\mathrm{Cr}$ & IT & - & $\mathrm{H}$ \\
\hline $\mathrm{He}$ & IT (End.) & - & $\mathrm{H}$ \\
\hline $\mathrm{He}$ & IT (End.) & - & $\mathrm{H}$ \\
\hline $\mathrm{Ph}$ & IT & $\mathrm{Vu}$ & $\mathrm{T}$ \\
\hline $\mathrm{He}$ & IT (End.) & $\mathrm{Vu}$ & $\mathrm{H}$ \\
\hline Th & IT,SS & - & $\mathrm{H}$ \\
\hline $\mathrm{He}$ & IT (End.) & LR & $\mathrm{H}$ \\
\hline $\mathrm{Ch}$ & IT & - & $\mathrm{H}$ \\
\hline $\mathrm{Ch}$ & IT & LR & $\mathrm{H}$ \\
\hline $\mathrm{He}$ & IT,ES & - & $\mathrm{H}$ \\
\hline $\mathrm{He}$ & IT, ES & - & $\mathrm{H}$ \\
\hline
\end{tabular}




\begin{tabular}{|c|c|c|c|c|c|c|}
\hline 159 & $\begin{array}{l}\text { Solanacea } \\
\text { Hyoscyamus nigrum }\end{array}$ & $\mathrm{He}$ & IT & LR & $\mathrm{H}$ & $\mathrm{P}$ \\
\hline 160 & $\begin{array}{l}\text { Lycium depressum Stocks. } \\
\text { Tamaricaceae }\end{array}$ & $\mathrm{Ph}$ & IT & . & $\mathrm{T}$ & $\mathrm{P}$ \\
\hline 161 & $\begin{array}{l}\text { Tamarix ramosissima Ledeb. } \\
\text { Thymelaeaceae }\end{array}$ & $\mathrm{Ph}$ & IT, ES & - & $\mathrm{T}$ & $\mathrm{P}$ \\
\hline 162 & $\begin{array}{l}\text { Dendrostellera lessertii (Wikstr.) Van Tigeh } \\
\text { Zygophyllaceae }\end{array}$ & $\mathrm{Ch}$ & IT & - & $\mathrm{H}$ & $\mathrm{P}$ \\
\hline 163 & Peganum harmala $\mathrm{L}$ & $\mathrm{He}$ & IT, ES, M & - & $\mathrm{H}$ & $\mathrm{P}$ \\
\hline 164 & Zygophyllum fabago $\mathrm{L}$. & $\mathrm{He}$ & IT & LR & $\mathrm{H}$ & $\mathrm{P}$ \\
\hline
\end{tabular}

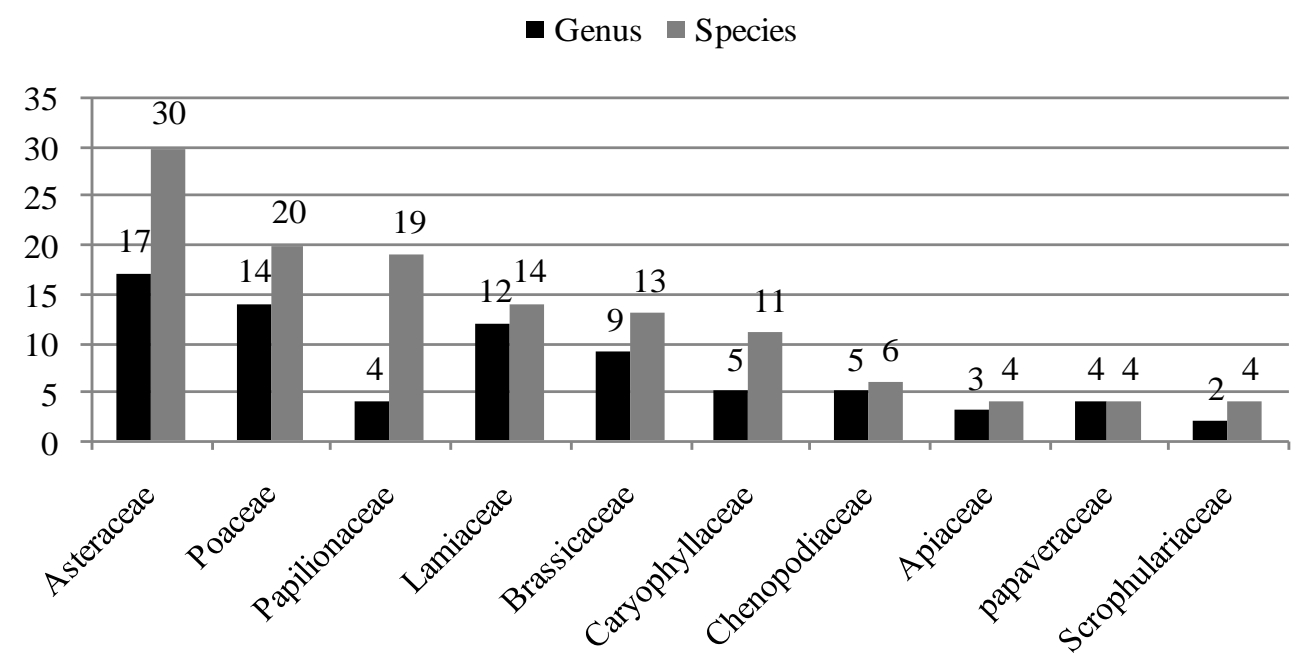

Figure 2. The large ten families in terms of the number of genera and species in the area

\section{Biological spectrum}

Hemicryptophytes were the dominant life forms accounting for 50\% (82 species) of all species in the area, followed by chamaephytes (25.6\%), therophytes (14.6\%), cryptophytes $(6.1 \%)$, and phanerophytes $(3.7 \%)$. The comparison of the floristic lifeform spectrum and Raunkiaer's normal spectrum pointed out a statistically significant difference between them $(p<0.05)$. The observed proportions were higher than expected for the chamaephytes, hemicryptophytes, and therophytes, and they were lower for the phanerophytes. Phanerophytes, followed by chamaephytes and hemicryptophytes, had the highest individual values determined from the $\chi^{2}$ test (Table 2).

Table 2. Comparison among life-form spectra and Raunkiaer's normal spectra in the Meymeh region (For Abbreviation, see Table 1).

\begin{tabular}{c|c|c|c|c|c|c}
\hline Life- form & Ph & Ch & He & Cr & Th & Total \\
\hline Number of species & 6 & 42 & 82 & 10 & 24 & 164 \\
Percentage of plant species & 4 & 26 & 50 & 6 & 14 & 100 \\
Raunkiaer's normal spectrum (\% of species) & 46 & 9 & 26 & 6 & 13 & 100 \\
Percentage Deviation & -42 & 17 & 24 & 0 & 1 & - \\
$\chi^{2}$ & 38.35 & 32.11 & 22.15 & 0.00 & 0.08 & 92.69 \\
\hline
\end{tabular}


The comparison of the life form spectrum of the case study with different studies in the Irano-Turanian growth zone is presented in Table 3. There were some differences and likenesses between the studies. The results of the $\chi^{2}$ test and correlation analysis demonstrated a significant correlation between the life-form spectrum in the area and those of other studies conducted in the vicinity of the area, particularly the Karkas hunting prohibited region $\left(\chi^{2}=8, P=0.001\right)$ and Yahya Abad region $\left(\chi^{2}=20.3, P=0.01\right)$.

Table 3. Comparison of different items in the study area and other studies conducted in Irano-Turanian Region (For Abbreviation, see Table 1).

\begin{tabular}{|c|c|c|c|c|c|c|}
\hline References & This study & $\begin{array}{l}\text { Batooli } e t \\
\text { al., } 2003\end{array}$ & $\begin{array}{l}\text { Abbasi } \text { et al., } \\
\quad 2012\end{array}$ & $\begin{array}{l}\text { Khajeddin } \\
\text { et al., } 2012\end{array}$ & $\begin{array}{c}\text { Yousofi } e t \\
\text { al., } 2011\end{array}$ & $\begin{array}{c}\text { Rahchamani } \\
\text { et al., } 2014\end{array}$ \\
\hline Location & Meymeh & Qazaan & Yahya Abad & karkas & Chadegan & Sarigol \\
\hline Elevation & $2004-3157$ & $1600-3550$ & $2000-2720$ & $1389-3880$ & $1950-3915$ & $1400-2840$ \\
\hline Annual rainfall (mm) & 177 & 181.5 & 147.22 & 240 & 324.3 & 273 \\
\hline Annual temperature $\left({ }^{\circ} \mathrm{C}\right)$ & 12 & 6.8 & 15.41 & 2.1 & 9.8 & 14 \\
\hline $\mathrm{Ph}$ & 4 & 10.8 & 1.6 & 7.9 & 5 & 7.4 \\
\hline $\mathrm{Ch}$ & 26 & 8.2 & 18.4 & 16.9 & 11 & 10.5 \\
\hline $\mathrm{H}$ & 50 & 35.4 & 44.7 & 51.8 & 44 & 33.9 \\
\hline $\mathrm{Cr}$ & 6 & 8.5 & 6.3 & 5.7 & 13 & 13.3 \\
\hline Th & 14 & 36.9 & 29 & 17.7 & 27 & 34.9 \\
\hline Total species & 164 & 398 & 190 & 278 & 339 & 498 \\
\hline$\chi^{2}$ with this study & 0.0 & 66.5 & 20.3 & 8.0 & 29.9 & 57.4 \\
\hline Pearson Correlation & 1.00 & 0.53 & $0.88^{*}$ & $0.96^{* *}$ & 0.81 & 0.56 \\
\hline
\end{tabular}

** Correlation is significant at the 0.01 level $(p<0: 001)$; *Correlation is significant at the 0.05 level $(p<0: 01)$.

\section{Chorological affinities}

About $74.4 \%$ of total plant species in the area belonged to the Irano-Turanian chorotype, whereas Irano Turanian-Euro Siberian, Irano Turanian-Mediterranean, and Irano Turanian-Euro Siberian-Mediterranean plant species contained 13.4\%, 5.5\%, and $2.4 \%$, respectively, of all plant species. Less than $4.3 \%$ of the total plant species belonged to other chorotypes (Table 4). The number of endemic species was 37 , and the endemism rate was $22.6 \%$.

Table 4. The Chorological affinities of plant species in the Meymeh region (For Abbreviation, see Table 1).

\begin{tabular}{l|c|c}
\hline \multicolumn{1}{c|}{ Chorotypes } & Number of species & Percentage of species \\
\hline $\begin{array}{l}\text { Monoregional } \\
\text { IT }\end{array}$ & 85 & 51.8 \\
Biregional & & \\
IT, ES & 22 & 13.4 \\
IT, M & 9 & 5.5 \\
IT, SA & 1 & 0.6 \\
IT,SS & 1 & 0.6 \\
Pluriregional & & \\
IT, ES, M & 4 & 2.4 \\
IT, SS, ES, M & 1 & 0.6 \\
IT,M,SS & 1 & 0.6 \\
Cosmopolitan & 3 & 1.8 \\
Endemic & 37 & 22.6 \\
$\quad$ Total & 164 & 100 \\
\hline
\end{tabular}




\section{IUCN categories}

The threatened species of the Meymeh region were evaluated on the basis of the criteria given by Jalili and Jamzad (1999) for assessing threat levels. Accordingly, 36 species $(22 \%)$ were situated in the different stages of the method. Ten and 26 species were ranked as vulnerable and lower risk, respectively. The herbaceous perennial species accounted for $72 \%$ (26 species) of the total threatened species, and 47.2\% (17 species) of them belonged to Irano-Turanian endemic species. Moreover, the families Asteraceae, Papilionaceae, and Poaceae were treated as the most important families as $16.7 \%$ (6 species) of their total species were allocated to the categories of threatened species (Fig. 3).

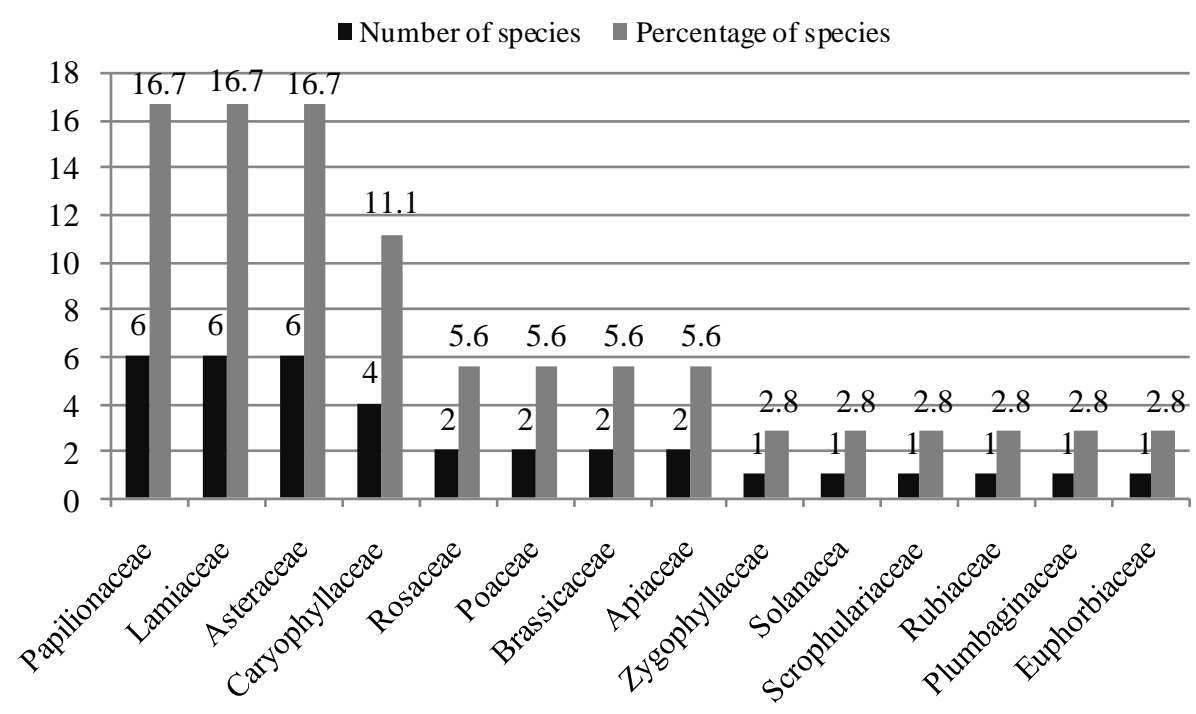

Figure 3. The most important families in terms of having the largest number of species in the categories of threatened species.

\section{Discussion}

The presence of 164 plant species belonging to 108 genera and 32 families indicates a considerable level of plant diversity in the study area. These species have formed diverse communities tailored to their ecological needs and the management imposed by humans over the past years. It seems that high plant diversity in the area is due to edaphic, topographic, and physiographic conditions. Of course, the climatic factor is also effective in this regard, but variations in climatic conditions of the area are less than the other factors (Khajeddin et al., 2012).

Asteraceae, Poaceae, and Papilionaceae were among the richest families in the area which is due to the high compatibility of these families with the arid and semiarid climate conditions. They are common compared to other plant families in the IranoTuranian growth zone (Aghaei et al., 2013; Jafari et al., 2016), especially in the central region of Iran (Yousofi et al., 2011; Abbasi et al., 2012; Khajeddin et al., 2012). In addition, the high frequency of Asteraceae may be related to the high percentage of vegetation destruction in the area (Archibold, 1995). Compared with the nearby Karkas hunting-prohibited region (Khajeddin et al., 2012), there are fewer 
species of flora in the Meymeh region. This is probably due to the higher habitat diversity along the Karkas Mountains.

In this study, the main fraction of species composition was Artemisia aucheri/Artemisia sieberi gradient. Distribution of these two species was contrasting, as Artemisia sieberi was the most dominant species at low altitude and that was gradually replaced by Artemisia aucheri at higher altitude. This finding is in agreement with the results of study conducted by Mousaei Sanjerehei et al., (2013), in which it was shown that distribution of these two species is associated to the complex environmental variations along the altitudinal gradient.

According to Raunkiaer's system, the life-form composition in the Meymeh region was dominated by hemicryptophytes followed by chamaephytes and therophytes in succession. The low percentages of cryptophytes and phanerophytes show that they are not adapted to climatic conditions in the region. A comparison of the life-form spectra of the area with those in the Karkas hunting-prohibited region (Khajeddin et al., 2012) and pastural region of Yahya Abad (Abbasi et al., 2012) showed the same results. In all the studies conducted in the Irano-Turanian growth zone, hemicryptophytes and phanerophytes usually comprise the highest and lowest percentages of life forms, respectively (Table 3 ). Hemicryptophytes are a large and complex life-form group with the resting bud at or near the soil surface. The frequency of the life form in the flora of the area is characteristic of the cool steppe region (Cain, 1950) and also indicates the dominance of cold and mountainous climates (Arcihold, 1995). Furthermore, the high percentage of chamaephytes characterized the colder climate and high altitude (Braun-Blanquet, 1932). Chamaephytes are often protected in the unfavorable season by fallen leaves or by the dense growth of the plant itself. In this life form, the species that provide the greatest protection of the buds are undoubtedly the very compact cushion plants such as Astragalus spp, Acanthophyllum spp, and Acantholimonsp spp in certain dry steppes (Cain, 1950). This type of plants was fairly common in the area, comprising about $15 \%$ (24 species) of all the available species. The remarkably high percentages of hemicryptophytes and chamaephytes $(76 \%$ for both) were also emphasized. Hemicryptophytes are always abundant in areas where chamaephytes are abundant, but they show no consistent trend (Cain, 1950).

Next in abundance were the therophytes, which indicate the existence of a heavy biotic pressure due to overgrazing and human interference. Low rainfall levels, high temperatures, consecutive droughts, a short growing season, and various factors of destruction such as overgrazing and agriculture were the most important factors for the increase of therophytes; this result was consistent with the results of studies conducted by Raunkiaer (1934) and Cain (1950), in which it was shown that the increase of therophytes reflects an effective strategy for avoiding water loss. Although therophytes occur abundantly in desert areas (Archibold, 1995), more or less, the high occurrence of the life form indicates there are some anthropogenic and overgrazing effects in the study areas (Grime, 2001; Ravanbakhsh et al., 2014).

Results of the $\chi^{2}$ test showed that Raunkiaer's normal spectrum was significantly different from the amount of phanerophytes, hemicryptophytes, and chamaephytes, while the differences between the amounts of cryptophytes and therophytes are not significant. The phanerophytes showed a maximum divergence from the normal spectrum. There was less of them than that of Raunkiaer's normal spectrum, which may be related to dryness of the region. This change seems much more significant 
and true to the climatic differences than those in other life forms, because the phanerophytes occur in the most favorable climates with high temperatures and humidity (Cain, 1950). The total number of chamaephytes was higher than that of Raunkiaer's normal spectrum. This finding can be related to the cold and mountainous conditions of the region and is in agreement with the results reported by Cain (1950) that demonstrated the positive correlation of this class' percentage with increasingly high altitudes, especially in areas with such widely diverse vegetation types as certain steppes. The hemicryptophytes reached a maximum in the area, where they were at about double the normal spectrum. This finding shows that they are adapted to the cold steppe climate of the area. Many species belonging to the Irano-Turanian phytogeographic region were found in this study, indicating that the study area belongs to this growth zone. In the flora of adjoining areas and even neighboring countries such as Turkey (Vural, 2005), Pakistan and Afghanistan (Rechinger, 1963-2010), Irano-Turanian elements compose a large proportion of the total species (Akhani, 2006), which represents the homogeneity of this growth zone (Abbasi et al., 2012).

The results of the assessment of threatened species showed that most belonged to herbaceous perennial species, which is consistent with the results reported in Jalili and Jamzad (1999) that demonstrated that herbaceous perennial species accounted for $71 \%$ of the total threatened species in Iran. Also, the same results were also obtained in Karkas hunting prohibited region (Khajeddin et al., 2012). Various factors caused the increase in numbers of vulnerable species in the area. Overgrazing was a major cause which led to the destruction of Astragalus schistosus, Poterium sanguisorba, Ferula ovina and Bromus tomentellus habitats. In contrast, finite population and low natural reproduction were determined to be the factors most effective on the vulnerability of Astragalus scleroclodus and Salvia eremophila. Cousinia piptocephala and Zataria multiflora the endemic species of the Irano-Turanian growth zone that established in individual habitats in terms of edaphic and biological characteristics. The most important factor that caused the deterioration of these species was human activity, such as overexploitation of the plant and land use change.

\section{Conclusion}

This study supports the general hypotheses; tests demonstrated that plant communities in areas that have a cold steppe climate with dry summers and relatively wet winters in the central plateau of Iran would have a high proportion of hemicryptophyte species belonging to the Irano-Turanian growth zone.

Besides the variations in species composition, the composition of life forms reflects the response of vegetation to variations in certain environmental factors. In this study, the dominance of hemicryptophytes, chamaephytes, and therophytes over other life forms seems to be a response to the cold steppe climate, high altitude, and human activities. Regardless of the type of ecosystem, it was noted that overgrazing in some parts of the area, especially in the lowlands, had led to the occupation of the area by invasive therophytes, indicating hyper-degradation. Therefore, overgrazing is viewed as a major cause of the deterioration of vegetation in the area. The best solution for sustainable management of the area would be to comply with the proper principles of range management aimed at reducing the intensity of grazing and prolonging exclosure. 
This study provides fundamental data about the flora of the area by means of a thorough botanical inventory. These findings will have special significance for further ecological research and for recommendations of proper guidance for the management, reclamation, and development of the area and other similar regions.

\section{REFERENCES}

[1] Abbasi, S., Afsharzadeh, S., Mohajeri, A. (2012): Study of flora, life forms and chorotypes of plant elements in pastural region of Yahya Abad (Natanz). - Iranian Journal of Plant Biology 11(4): 1-12.

[2] Aghaei, R., Alvaninejad, S., Zolfaghari, R.,Gharehlar, M.R.M. (2013): Flora, life form and geographical distribution of plants in West-South forests of Iran (Case study: Vezg, Yasouj). - International Journal Farming and Allied Sciences 2(23): 1046-1053.

[3] Akhani, H. (2006): Flora Iranica: facts and figures and a list of publications by K.H. Rechinger on Iran and adjacent areas. - Rostaniha 7: 19-61.

[4] Archibold, O.W. (1995): Ecology of world vegetation. - Chapman and Hall Inc, London.

[5] Asri, Y. (2003): Plant Diversity in Touran Biosphere Reservoir. - Research Institute of Forests and Rangelands press, Tehran.

[6] Batooli, H. (2003): Biodiversity and species richness of plant elements in Qazaan reserve of Kashan. - Pajouheh \& Sazandegi 61: 85-103.

[7] Braun-Blanquet, J. (1932): Plant sociology. - Macgran- Hill, New York/London.

[8] Cain, S.A. (1950): Life-forms and phytoclimate. - The Botanical Review 16(1): 1-32.

[9] Davis, P.H. (1965-1988): Flora of Turkey and the East Aegean Island. - Edinburgh University Press, London.

[10] Djamali, M., Brewer, S., Breckle, S.W., Jackson, S.T. (2012): Climatic determinism in phytogeographic regionalization: A test from the Irano-Turanian region, SW and Central Asia. - Flora 207: 237-249.

[11] Ghahraman, A. (1975-2005): Colored Flora of Iran. - Research Institute of Forests and Rangelands Press, Tehran.

[12] Ghahreman, A., Attar, F. (1999): Biodiversity of Plant Species in Iran. - Vol. 1, Tehran University press, Iran.

[13] Grime, J.P. (2001): Plant strategies, vegetation processes and ecosystem properties. John Wiley and Sons Inc, New York.

[14] IUCN. (1981): How to use the IUCN Red Data Book Categories. Threatened plants Committee Secretariat. - IUCN, Kew.

[15] Jafari, Z., Kianian, M.K., Aslani, F., Akbarlo, M. (2016): Floristic Composition, Life Forms and Geographical Distribution (Case Study: Lashgardar Rangelands of Malayer, Iran). - Journal of Environmental Science and Technology 9 (1): 140-148.

[16] Jalili, A., Jamzad, Z. (1999): Red data book of Iran. - Research institute of Forests and Rangelands. Tehran.

[17] Khajeddin, S.J., Yeganeh, H. (2012): The flora, life form and endangered species of karkas hunting prohibited region, Isfahan, Iran. - Iranian Journal of Biology 25 (1): 7-20.

[18] Mabberley, D.J. (2008): The Plant- Book, Aprotable dictionary of Higher Plants. Cambridge University Press.

[19] Mobayen, S. (1975-1996): Flora of Iran, Vascular Plants. - Vol. 1-4, Tehran University Press, Iran.

[20] Moradi, G.h., Marvie, M.R., Zahedi, G.h., Shirvany, A., Zargham, N. (2010): Life form and geographical distribution of plants in Posthband region, Khonj, Fars Province, Iran. Journal of Forestry Research 21(2): 201-206. 
[21] Mousaei Sanjerehei., M. (2012): Soil-Vegetation Relationships in Arid Rangelands (Case Study: Nodushan Rangelands of Yazd, Iran). - World Academy of Science, Engineering and Technology 6: 7-23.

[22] Muller-Dombois, D., Ellenberg, H. (1974): Aims and Methods of Vegetation Ecology. John Wiley and Sons, New York.

[23] Nimais, P.L. (1985): Structure and floristic composition of high-arctic tundra: NyAlesund (Svalbard Archipelag). - Nordic Journal of Botany 17: 47-58.

[24] Rahchamani, N., Ezazi, A., Ghahremaninejad, F. (2014): The flora of Sarigol National Park, Northern Khorassan Province. - Journal of Biodiversity and Environmental Sciences 4 (6): 278-307.

[25] Ramak Masoumi, A. (1986-2000): Astragalus communities of Iran. - Vol. 1-4, Publishing Research Institute of Forests and Rangelands Press, Tehran.

[26] Raunkiaer, C. (1934): The life forms of plants and statistical geographical. - Oxford: Clarendon Press.

[27] Ravanbakhsh, M., Amini, T. (2014): A Study on Floristic Composition, Chorology and Ecological Structure: A case Study from a Small-scale Forest Reserve, Talesh, Iran. IUFS Journal of Biology 73(1): 43-51

[28] Rechinger, K.H. (1963-2010): Flora Iranica. - Vol. 1-178. Akademische Druck-U, Verlagsanstalt, Graz. Austria.

[29] Vural, C. (2005): The Flora of Erciyes DaÛÝ (Kayseri, Turkey). - Turkish Journal of Botany 29: 185-236.

[30] Yousofi, M., Safari, R., Nowroozi, M. (2011): An investigation of the flora of the Chadegan region in Isfahan province. - Journal of Plant Biology 3(19): 75-96.

[31] Zohary, M. (1963): On the geobotanical structure of Iran. - Weizmann Science Press of Israel, Jerusalem.

[32] Zohary, M. (1973): Geobotanical Foundation of the Middle-East. - Vol 1-2. Department of Botany, Gustav Fischer Verlag, Stuttgart. 